associated with decreased HROOL include: comorbidity, older age at transplantation, medication side effects, and parental conflict. Two specific problems in pediatric organ transplantation are adherence to medication and transition from pediatric to adult transplant care. Early disease onset, poor nutritional status, growth deficits, and longer duration of illness prior to transplant have been identified as factors contributing to an adverse cognitive development of these children.

Studies are heterogeneous regarding operationalization of HRQOL, study design, length of follow-up, and age of the children. There are only few prospective multi-center studies, which should be encouraged in future research including specific internationally accepted validated instruments.

Against the background of a new era of immunosuppressive therapy (steroid minimization, individualized therapy), a better long-term outcome in these children could be expected.

\section{CHILDREN AS DONORS: A NATIONAL PEDIATRIC INTENSIVE CARE STUDY TO ASSESS PROCUREMENT OF ORGANS AND TISSUES}

doi:10.1136/archdischild-2012-302724.0161

MJ Siebelink, MJIJ Albers, PF Roodbol, HBM van de Wiel. University of Groningen, University Medical Center Groningen, Groningen, The Netherlands

Objectives Shortage of size-matched organs and of tissues is the key factor limiting transplantation in children. Empirical data on the procurement process in children is sparse. This study aimed to gain insight into the recognition of potential pediatric donors in the Netherlands and the procurement process.

Methods A national retrospective cohort study in the Dutch pediatric intensive care units. The records of 683 deceased children were analyzed by two independent donation experts and procurement process data were compared with the national protocol.

Results From 2003 thru 2006, 74 (11\%) of the deceased children were found to have been suitable for organ donation and 132 (19\%) for tissue donation. Sixty-two (84\%) potential organ donors had been correctly identified; parental consent had been obtained and donation effectuated in 26/62 children (42\%). Sixty-three potential tissue donors (53\%) had been correctly identified; parental consent had been obtained and donation effectuated in $17 / 63$ children (27\%). Conclusion Recognition of pediatric organ donors by medical professionals is acceptable; recognition of tissue donors may be improved. Efforts to address the shortage of organs and tissues for transplantation in children should focus on the gap between recognition of donors and parental consent. We suggest such studies should not only assess the process itself, i.e. the competencies of the professional staff (micro-level) but also the influence of legislation, societal views on donation by children, and the potential relevance of children's views on donation (macro-level).

\section{CONGENITAL CHLORIDE DIARRHEA: A SINGLE CENTRE EXPERIENCE WITH 43 CHILDREN}

doi:10.1136/archdischild-2012-302724.0162

'SM Shabib, ${ }^{2}$ Al Al Mehaedib, ${ }^{1} \mathrm{HM}$ Nazer, ${ }^{1} \mathrm{AA}$ Abbad. ${ }^{1}$ Pediatrics; ${ }^{2}$ King Faisal Specialist Hospital \& Research Centre, Rivadh, Saudi Arabia

Background Congenital chloride diarrhea(CCD), A rare deadly autosomal recessive disorder of chronic diarrhea in infancy.

Methods Patients diagnosed with CCD between1986-2009 were studied. The demographic data, clinical findings and biochemical findings were collected and statistically analyzed.

Results Forty-three patients $(28 \mathrm{M} / 15 \mathrm{~F})$ had CCD. Fifteen patients $(35 \%)$ were diagnosed after one year of age (late referral or misdiagnosis as Bartter syndrome). Premature delivery in 24 cases (55.8\%). Polyhydramnios in 26 pregnancies. All patients were distributed among 19 families with 33 children being the outcome of consanguineous marriages. Intractable diarrhea was the presenting symptom in 40 patients (93\%), Biochemical data revealed: Serum potassium (1.3-4.1, mean $2.4 \mathrm{Mmol} / \mathrm{l})$, s. chloride (39-95, mean76.2Mmol/l), s.bicarbonate (22-54) meam-37.6 Mmol/). Fecal chloride (134 \pm 21.6 , mean \pm SD) (range 90-205). The fecal chloride over fecal sodium plus potassium ratio was $0.6(1.1 \pm 0.3$, mean $\pm \mathrm{SD})$ $(\mathrm{N} .=0.2)$. Associated disorders were: chronic renal failure $7(16 \%)$, congenital anomalies 8 (19\%), mental retardation4 (9.3\%) seizures 8 $(19 \%)$, and brain atrophy 4 (9\%). Complications were seen mostly among patients with late referral or poor compliance. At diagnosis, $35(81.4 \%)$ cases were below -2SD for weight for-age, 31 (72\%) for weight-for-height, and 31 (72\%) for height-for-age. Children under five years of age showed improvement in weight for height as compared with older children.

Conclusions CCD is a treatable cause of intractable diarrhea in infancy.

Awareness, early diagnosis and proper management are essential in preventing irreversible and long-term organ damage and a better outcome compared to those with late referrals.

CCD is to be considered in infants with severe persistent diarrhea where a high rate of consanguineous marriage prevails.

\section{LIVING DONOR LIVER TRANSPLANTATION FOR ALAGILLE SYNDROME: RECIPIENT CHARACTERISTICS AND OUTCOME IN A SINGLE CENTER}

doi:10.1136/archdischild-2012-302724.0163

MM Tiao', IF Huang'ㄹ. Liver Transplantation Team. 'Pediatrics, Kaohsiung Chang Gung Memorial Hospital and Chang Gung University College of Medicine; ${ }^{2}$ Pediatrics, Kaohsiung Veterans General Hospital, Kaohsiung, Taiwan R.O.C.

Background and aims Alagille syndrome (AGS) is rare in Taiwan. The aim of this study was to review our institutional experience with liver transplantation (LT) for AGS.

Methods We performed a retrospective analysis of transplant records of patients diagnosed as AGS and underwent LT between 1987 and 2010. Nine patients underwent living donor LT.

Results Cholestasis and characteristic faces were seen in all patients. Posterior embryotoxon was seen in 4/9 (44.4\%), butterfly vertebrae in $3 / 9(33.3 \%)$, heart defect (pulmonary stenosis in 2 ) in $3 / 9(33.3 \%)$, and renal disease in $2 / 9(22.2 \%)$ patients. Iminodiacetic acid scans showed no excretion of isotope into the bowel after 24 hours in $4 / 9(44.4 \%)$. A small gallbladder on ultrasonography was noted in $3 / 9(33.3 \%)$ and suggested a false diagnosis of biliary atresia. All underwent diagnostic laparotomy and liver biopsy. Liver biopsy showed characteristic features of paucity of interlobular bile ducts in all patients. Kasai portoenterostomy was not performed in any patient before being referred for LT. The mean age at time of LT was 4.6 years. The 5 -year overall survival rate after living donor LT was $88.9 \%$.

Conclusions Our conclusion is that the clinical features of AGS are informative. Histological confirmation is important in the diagnosis. These findings support the concept that infants with liver diseases warrant early referral to a specialist center.

\section{LINEAR GROWTH AFTER PEDIATRIC LIVER TRANSPLANTATION}

doi:10.1136/archdischild-2012-302724.0164

W Saleem, H Abdelrahman, A Elawwa, A Soliman. Pediatrics, Hamad Medical Center, Doha, Qatar

To determine growth patterns in a children undergoing liver transplantation, the outcomes of orthotopic liver transplantations performed in 10 children at Hamad General Hospital between October 2005 and October 2009 were reviewed. The mean age at transplantation was $27+/-30$ months; $80 \%$ of the children were females. The 